\title{
The TF-miRNA Coregulation Network in Oral Lichen Planus
}

\author{
Yu-Ling Zuo, ${ }^{1}$ Di-Ping Gong, ${ }^{2}$ Bi-Ze Li, ${ }^{3}$ Juan Zhao, ${ }^{4}$ Ling-Yue Zhou, ${ }^{4}$ \\ Fang-Yang Shao, ${ }^{2}$ Zhao Jin, ${ }^{5}$ and Yuan $\mathrm{He}^{2}$ \\ ${ }^{1}$ Teaching Hospital of Chengdu University of Traditional Chinese Medicine, Shierqiao Road 39, Chengdu, Sichuan 610072, China \\ ${ }^{2}$ Laboratory of Oral Biomedical Science and Translational Medicine, School of Stomatology, Tongji University, \\ Middle Yanchang Road 399, Shanghai 200072, China \\ ${ }^{3}$ Preclinical Medicine College, Chengdu University of Traditional Chinese Medicine, Shierqiao Road 37, Chengdu, \\ Sichuan 610075, China \\ ${ }^{4}$ Acupuncture and Tuina College, Chengdu University of Traditional Chinese Medicine, Shierqiao Road 37, Chengdu, \\ Sichuan 610075, China \\ ${ }^{5}$ College of Basic Medicine, Chengdu University of Traditional Chinese Medicine, Shierqiao Road 37, Chengdu, \\ Sichuan 610075, China
}

Correspondence should be addressed to Zhao Jin; dr.jinzhao@163.com and Yuan He; drheyuan@tongji.edu.cn

Received 8 September 2014; Revised 4 December 2014; Accepted 8 December 2014

Academic Editor: Tatsuya Akutsu

Copyright (C) 2015 Yu-Ling Zuo et al. This is an open access article distributed under the Creative Commons Attribution License, which permits unrestricted use, distribution, and reproduction in any medium, provided the original work is properly cited.

Oral lichen planus (OLP) is a chronic inflammatory disease that affects oral mucosa, some of which may finally develop into oral squamous cell carcinoma. Therefore, pinpointing the molecular mechanisms underlying the pathogenesis of OLP is important to develop efficient treatments for OLP. Recently, the accumulation of the large amount of omics data, especially transcriptome data, provides opportunities to investigate OLPs from a systematic perspective. In this paper, assuming that the OLP associated genes have functional relationships, we present a new approach to identify OLP related gene modules from gene regulatory networks. In particular, we find that the gene modules regulated by both transcription factors (TFs) and microRNAs (miRNAs) play important roles in the pathogenesis of OLP and many genes in the modules have been reported to be related to OLP in the literature.

\section{Introduction}

Oral lichen planus (OLP) is a chronic inflammatory disease that acts on mucous membranes inside the mouth and causes bilateral white lacy patches or plaques on the buccal mucosa, tongue, and gingivae [1]. It is found that OLP affects $0.5 \%$ to $2 \%$ of the adult population, especially the adults over 40 years old, where OLP tends to affect women rather than men with a ratio about $1.4: 1[2,3]$. Compared with cutaneous lichen planus, oral lichen planus lesions are more difficult to be treated with frequent recurrence. Furthermore, OLP may be at risk of developing into oral cancer as the result of carcinogenic exposures, where the erosive OLP lesions might be more sensitive to carcinogens than normal oral mucosa [1]. Currently, oral cavity cancer has become one of the 10 most frequently diagnosed cancers with increasing mortality in East Europe $[4,5]$. However, the pathogenesis of OLP and how it is developed into oral cancer is still unclear $[6,7]$. Therefore, it is extremely urgent to pinpoint the molecular mechanisms underlying the pathogenesis of OLP so that accurate diagnosis can be made and effective therapies can be developed.

Recently, the accumulation of large amount of omics data, especially transcriptome data, provides opportunities to identify the molecules related to diseases. Accordingly, many works have investigated OLPs with transcriptome data. For example, the genes CCR5, CD14, and beta-catenin have been identified to play important roles in the pathogenesis of OLP $[8,9]$. Moreover, Tao et al. identified some genes that are differentially expressed in OLPs, such as FOXP3, ANGPT1, and MMP1, and these genes may be related to the development of OLP [10]. In general, the above-mentioned studies assume 
those differentially expressed genes between OLPs and controls are related to OLP. However, the differentially expressed genes are usually treated independently, which is actually not the case. It has been found that complex diseases, for example, cancers, happen due to the dysregulation of functional gene sets or molecular pathways [11, 12]. In other words, the genes involved in the same disease tend to have functional relationships. Therefore, it is necessary to investigate disease related genes from a systematic perspective. Except for the above-mentioned genes, some small noncoding RNAs, that is, microRNAs (miRNAs), were found to play important roles in cancer by targeting oncogenes or tumor suppressor genes [13]. For example, mir-21 was found to be overexpressed in several tumor types [14], and let-7 inhibits lung tumorigenesis by repressing the expression of the RAS oncogene [15]. More recently, Gassling et al. found that the dysregulation of some miRNAs has important pathophysiological impacts on OLP [16]. For instance, mir-21, mir-181b, and mir-345 were found to be upregulated in OLPs and have critical roles in the malignant transformation of OLP to oral cancer.

In this work, we present a novel approach to identify gene modules that may play important roles in the pathogenesis of OLP by assuming that OLP is caused due to the dysregulation of certain gene modules. Furthermore, based on the gene modules as well as their transcription factor and miRNA regulators, we construct a TF-miRNA coregulation network. By investigating the genes and their regulators in the coregulation network, we find that some of them have already been reported to be related to OLP or oral cancer, indicating the important roles of the regulation network in OLP. In addition, we notice that the genes involved in the regulation network can serve as disease associated pattern and separate OLPs from controls very well, which is also validated by another independent real dataset, demonstrating the potential of the gene modules we identified as disease associated pattern and therapeutic targets.

\section{Materials and Methods}

2.1. Gene and miRNA Expression Data. The matched gene and miRNA expression data were obtained from the Gene Expression Omnibus (GEO) database [17]. Both mRNA (accession number: GSE38616) and miRNA (accession number: GSE38615) expression profiles were measured in 7 healthy individuals and 7 oral lichen planus patients [16]. To further validate the genes identified from the above datasets, another gene expression dataset (accession number: GSE52130) was retrieved from GEO, which was originally measured in 14 oral samples and 9 genital epithelium samples. Here, the gene expression profiles from the 14 oral samples consist of 7 normal oral samples and 7 oral lichen planus samples were kept for validation. All the three expression datasets have been preprocessed and normalized when we downloaded them, and the data were used in later sections without further preprocessing.
2.2. Identification of Differentially Expressed Genes and miR$N A s$. In general, the genes that are differentially expressed between diseases and controls are related to diseases to some extent. In this work, the genes that are differentially expressed between OLPs and controls were detected with Student's $t$ test. The genes with $P$ values less than 0.05 were regarded to be differentially expressed genes (DEGs), and the same for miRNAs. Consequently, 2587 differentially expressed genes (GSE38616) and 90 differentially expressed miRNAs (GSE38615) were obtained for further analysis.

\subsection{Identification of Network Modules Associated with OLP.} A gene coexpression network was constructed for OLPs based on their corresponding gene expression data, where one gene was linked to another if their coexpression measured with Pearson's correlation coefficient was significantly high ( $P$ value cutoff of 0.05 ) and the weights accompanying the edges were their corresponding correlation coefficients. Subsequently, network modules that consist of densely connected genes were detected with ClusterONE [18], and 154 network modules were detected here. Furthermore, after investigating the network modules, we merged two modules if more than one-third of genes from the smaller module occur in the larger one. As a result, 125 modules were kept for further analysis. For each network module, it will be regarded to be related to OLP if the module is enriched with the above identified differentially expressed genes, where the enrichment analysis was performed with Fisher's exact test ( $P$ value cutoff of 0.01 )

2.4. miRNA-Gene Regulations. The target genes of miRNAs were collected from both predictions and experimentally determined ones. For the predictions, several tools, including PicTar [19], miRanda [20], MicroT [21], and TargetScan [22], were employed to predict the target genes of miRNAs. Specifically, we picked up the interactions between genes and miRNAs predicted by at least two tools to avoid false positives. Moreover, the target information of miRNAs deposited in Tarbase [23] was obtained and merged with the predictions, where all the miRNA-gene interactions from Tarbase have been experimentally validated.

2.5. The TF-miRNA Coregulation Network in OLP. After obtaining the network modules, we first checked which miRNAs may regulate the network modules. Given a network module and a miRNA, the miRNA will regulate the module if its target genes are enriched in the modules with Fisher's exact test ( $P$ value cutoff of 0.01$)$. In particular, we only considered the differentially expressed miRNAs (DemiRs) here since these DemiRs are more likely related to OLP. Furthermore, given a network module, the transcription factors (TFs) that possibly regulate the modules were identified if these TFs belong to the module and coexpress with other genes in the module. Note that here we suppose the TFs that coexpress with other genes in the module will regulate the genes within the module. Consequently, we detected 6 network modules that are coregulated by TFs and miRNAs, where the modules are enriched with DEGs. We assumed that the TF-miRNA 


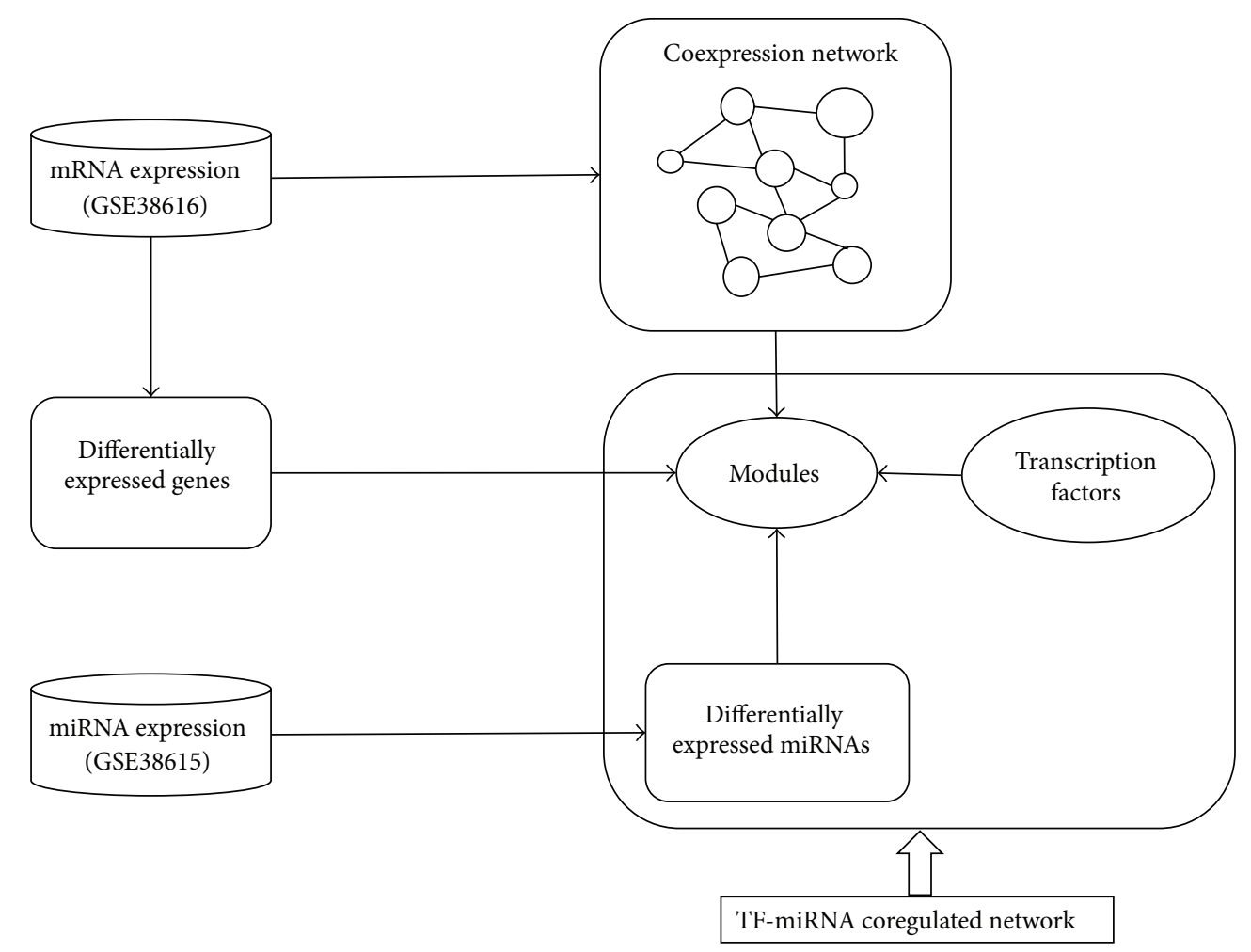

FIGURE 1: Schematic illustration of the pipeline to detect the TF-miRNA coregulation networks in oral lichen planus.

TABLE 1: The detailed information about the 6 network modules as well as their TF and miRNA regulators.

\begin{tabular}{lcccl}
\hline Module & \#Nodes & \#Edges & miRNAs & Transcription factors \\
\hline Module 1 & 134 & 952 & hsa-miR-628-5p & $\begin{array}{l}\text { SALL2, PEG3, ZNF865, HES3, ZNF283, ZIM2, } \\
\text { FOSB, PAX6, SIX2, ZNF616, KDM5D, SRY, RFX4, } \\
\text { and ZFY }\end{array}$ \\
Module 2 & 62 & 594 & $\begin{array}{c}\text { hsa-miR-595 } \\
\text { hsa-miR-34c-5p } \\
\text { hsa-miR-34a } \\
\text { hsa-miR-26b }\end{array}$ & ELK3, RFX8, MIXL1, HIF1A, and ZNF552 \\
Module 3 & 160 & 1301 & $\begin{array}{l}\text { hsa-miR-29a } \\
\text { hsa-miR-190 } \\
\text { hsa-miR-146b-5p }\end{array}$ & LIN28A \\
Module 4 & 80 & 405 & 335 & THRA, NR1D1, and NR1D2 \\
Module 5 & 55 & 241 & &
\end{tabular}

coregulation network which consists of the 6 modules plays important roles in the development of OLP.

\section{Results and Discussion}

Figure 1 depicts the flowchart of our proposed approach for identifying the TF-miRNA coregulation network in OLP, and we applied it to a real dataset which contains 7 healthy individuals and 7 OLP patients, where the dataset contains the matched gene (GSE38616) and miRNA (GSE38615) expression profiles. From the dataset, we detected 2587 differentially expressed genes (DEGs) and 90 differentially expressed miRNAs (DemiRs). We further constructed a gene coexpression network and identified modules that were coregulated by miRNAs and TFs (Figure 2). Table 1 lists the detailed information about the 6 network modules as well as their regulators.

3.1. The Network Modules Are Enriched with Oral Lichen Planus Related Genes. After getting the 6 network modules, we first investigated the genes in each module. By querying the PubMed, we found that 59 out of the 497 genes belonging to the 6 modules have been reported to be relevant to oral cancer. Here, for each network module, we gave examples 


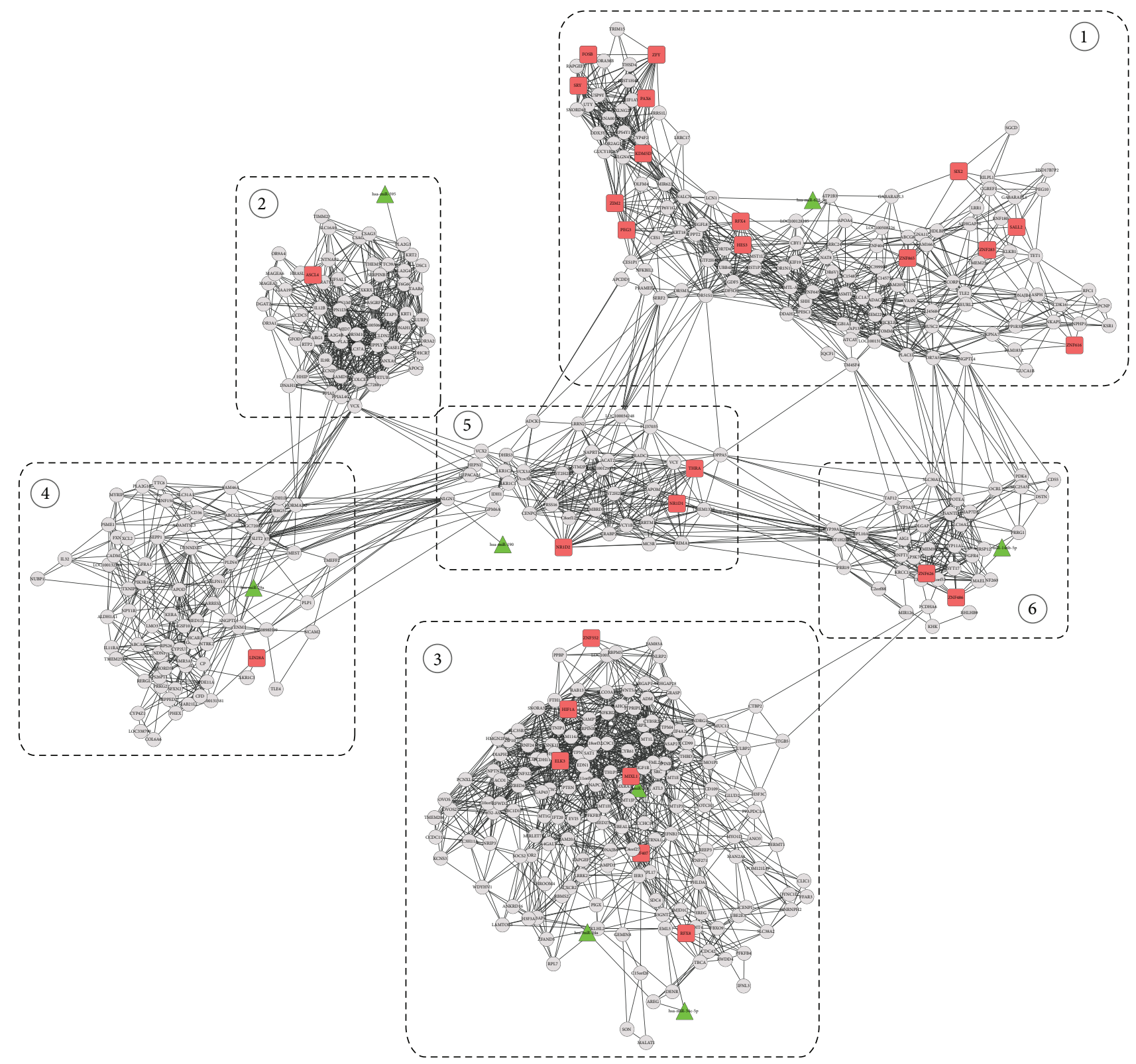

$\triangle$ miRNA
$\square$ Transcription factor
$\bigcirc$ Gene

FIGURE 2: The TF-miRNA coregulation network with 6 modules, where the green nodes represent differentially expressed miRNAs, red nodes denote the transcription factors, and gray nodes represent genes in modules, respectively.

about genes that have been reported to be related to OLP or oral cancer in the literature (Table 2). For example, it was reported that the gene KRT18 was related to tumor differentiation and metastasis and plays important roles in the malignant transformation of OLP to oral squamous cell carcinoma [24]. Another gene PTEN was reported to be downregulated in oral squamous cells, which in turn downregulates the expression of cyclin D1 and leads to the suppression of cell growth, indicating that targeting PTEN may help treat oral cancer [25]. Moreover, IGF1R has been reported to control cell proliferation of oral cancer [26, 27]. The overlap between known oral cancer associated genes and our identified module genes indicates that the genes belonging to these modules are related to OLP as well as its development to oral cancer.

Next, we investigated the functions of the network module genes. For each module, functional and pathway enrichment analyses were performed with DAVID [28], and the 
TABLE 2: Examples of genes from each network module that have been reported to be related to OLP or oral cancer.

\begin{tabular}{lcc}
\hline Network module & Gene symbol & PubMed IDs \\
\hline \multirow{3}{*}{ Module 1 } & KRT18 & $19575986 ; 22677743 ; 7527618 ;$ \\
& SHH & $11857543 ; 21945071 ; 21496886$ \\
& FOSB & $19653276 ; 15926923$ \\
\hline \multirow{2}{*}{ Module 2 } & MAGEA3 & $19187853 ; 12855658$ \\
& KRT1 & $20002980 ; 16334838 ; 10896780$ \\
& MAGEA6 & $18197853 ;$ \\
\hline \multirow{3}{*}{ Module 3 } & HIF1A & $19717330 ; 19449077 ; 18630523$ \\
& PTEN & $17067457 ; 15453811 ; 15805158$ \\
Module 4 & IGF1R & $17786320 ; 23106397 ; 19584075$ \\
\hline \multirow{2}{*}{ Module 5 } & CP & $23812204 ; 19884712 ; 17066447$ \\
& ABCG2 & $18429968 ; 15801936 ; 15618737$ \\
& ALDH1A1 & $22725270 ; 22782852 ; 21441790$ \\
\hline \multirow{3}{*}{ Module 6 } & IDH1 & $22385606 ; 21383741 ; 19378339$ \\
& CRABP2 & $19197536 ; 16568407 ; 11437413$ \\
\hline
\end{tabular}

detailed results can be found in Table 3, where only the processes we thought related to OLP were listed for clarification. From the analysis, we can see the biological processes in which the modules involved are related to the initiation and development of OLP. For example, it was reported recently that the pathogenesis of OLP was associated with some systemic diseases that can cause midbrain injuries [29]. The inhibition of phospholipase A2 activity that is associated with numerous inflammatory processes was found to be related to the mechanism of OLP [30]. The sensory perception, such as anxiety and tension, has been reported to be an important factor in the development of OLP [31]. It was reported that oral lichen planus can be caused by a variety of stimuli and the preservation of keratin in oral mucosa was an efficient way for the treatment of the disease [32]. Compared with normal controls, the OLPs tend to have increased angiogenesis, indicating OLP is associated with the induction of aberrant angiogenesis [7]. In addition, the symptoms of OLP are always accompanied by compromised wound healing [33], and the epidermal growth factor receptors were found to be significantly higher in OLPs [34].

Except for biological processes, functional enrichments analysis implies that some molecular functions, such as cytosol, steroid hydroxylase activity, and oxidoreductase activity, also have important impacts on OLPs. Considering that OLP is often treated with steroids and vitamin A analogues [35], it is not surprising that steroid hormone receptor activity, retinoid metabolic process, and vitamin A metabolic process are enriched in our identified modules. Moreover, the metabolism of xenobiotics by cytochrome $\mathrm{P} 450$ has been reported to result in the oral and pharyngeal cancers [36]. In addition, it was found that the metallic ion content can increase the damage to the oral mucosa cells [37], which is consistent with the enrichment of the iron ion homeostasis and binding.

From the analysis of the genes belonging to our identified modules, we can see that these modules are indeed related to the development of OLP. In addition, we identified some important biological processes that have important roles in the development of OLP, such as the metabolism of xenobiotics by cytochrome P450 and vitamin A metabolic process. The detailed information about the biological processes in which the TFs and miRNAs are involved can be found in Supplementary Table I available online at http://dx.doi.org/10.1155/2015/731264.

3.2. miRNAs Regulators of Network Modules Are Associated with OLP. In the TF-miRNA regulation network, there are in total 8 miRNAs, which were picked up from the 90 differentially expressed miRNAs. Among the 8 miRNAs, some of them have been reported to be related to OLP or oral cancer in the literature. For example, miR-26b was found to be significantly low expressed in OLP lesions compared with controls [38], miR-29a was remarkably differentially expressed in the oral squamous cell carcinoma metastasis [39], and miR-628 was able to discriminate hand-footmouth diseases from healthy controls [40]. According to the Human microRNA Disease Database (HMDD) [41], a manually curated disease-miRNA association database, mir$146 \mathrm{~b}$ was reported to be associated with diverse neoplasms including oral cancer. In addition, two of the 8 DemiRs, that is, hsa-miR-146b-5p and hsa-miR-26b, have been reported previously to be related to OLP [16].

Furthermore, we derived the interactions between miRNAs and target genes from the 6 modules in TF-miRNA coregulation network. Figure 3 shows the regulation network composed of miRNAs and target genes. By investigating the expressions of miRNAs and their target genes, we noticed that the expressions of 4 miRNAs, hsa-miR-190, hsa-miR146b-5p, hsa-miR-29a, and hsa-miR-595, were negatively correlated with that of their target genes, which is consistent with the observation that miRNAs generally repress the expression of their target genes. Interestingly, these four miRNAs were highly expressed in OLP while their target genes were downregulated. The detailed information about the expression levels of miRNAs and their target genes in modules can be found in Supplementary Table II.

\subsection{Transcription Factors Regulating the Network Modules} Are Associated with OLP. We also investigated the 27 transcription factors involved in the TF-miRNA coregulation network. By querying the PubMed, some TFs were found already reported to be related to OLP or oral cancer. For example, in module 3 , the transcription factor HIF1A is a master transcriptional regulator of the adaptive response to hypoxia. It was found that RTP801 and VEGF, the target genes of HIF1A, were significantly low expressed in OLPs [42]. The transcription factor LIN28A from module 4 has been reported to regulate cancer stem cell-like properties and can act as an appropriate target for oral squamous cell carcinoma treatment [43]. In module 1, AX6 was found to 
TABLE 3: Functional enrichment analysis of genes in network modules.

\begin{tabular}{|c|c|c|}
\hline $\begin{array}{l}\text { Network } \\
\text { module }\end{array}$ & Enriched functions related to OLP & $P$ value $(<0.05)$ \\
\hline \multirow{6}{*}{ Module 1} & GO:0030901 midbrain development & 0.00337086 \\
\hline & $\begin{array}{l}\text { GO: } 0016702 \sim \text { oxidoreductase activity, acting on single donors } \\
\text { with incorporation of molecular oxygen, incorporation of two } \\
\text { atoms of oxygen }\end{array}$ & 0.00560592 \\
\hline & GO:0008285 negative regulation of cell proliferation & 0.01400315 \\
\hline & GO:0007600 sensory perception & 0.032006 \\
\hline & GO:0007435 salivary gland morphogenesis & 0.0376765 \\
\hline & GO:0002052 positive regulation of neuroblast proliferation & 0.04294326 \\
\hline \multirow{3}{*}{ Module 2} & GO:0004623 phospholipase A2 activity & 0.00233 \\
\hline & GO:0050877 neurological system process & 0.00764739 \\
\hline & GO:0051606 detection of stimulus & 0.04745557 \\
\hline \multirow{6}{*}{ Module 3} & GO:0001525 angiogenesis & 0.00589117 \\
\hline & GO:0005829 cytosol & 0.00679876 \\
\hline & GO:0042060 wound healing & 0.01651834 \\
\hline & GO:0045740 positive regulation of DNA replication & 0.01978034 \\
\hline & $\begin{array}{l}\text { GO:0007173 epidermal growth factor receptor signaling } \\
\text { pathway }\end{array}$ & 0.01978034 \\
\hline & GO:0015629 actin cytoskeleton & 0.04636455 \\
\hline \multirow{4}{*}{ Module 4} & GO:0006879 cellular iron ion homeostasis & 0.00604241 \\
\hline & GO:0000041 transition metal ion transport & 0.029349054 \\
\hline & GO:0055114 oxidation reduction & 0.03217 \\
\hline & hsa00980:Metabolism of xenobiotics by cytochrome $\mathrm{P} 450$ & 0.03978611 \\
\hline \multirow{4}{*}{ Module 5} & GO:0003707 steroid hormone receptor activity & 0.00495459 \\
\hline & GO:0006766 vitamin metabolic process & 0.01044366 \\
\hline & GO:0001523 retinoid metabolic process & 0.04657368 \\
\hline & GO:0006776 vitamin A metabolic process & 0.04657368 \\
\hline \multirow{3}{*}{ Module 6} & GO:0008395 steroid hydroxylase activity & 0.02851299 \\
\hline & GO:0005887 integral to plasma membrane & 0.02900047 \\
\hline & GO:0008202 steroid metabolic process & 0.04570667 \\
\hline
\end{tabular}

regulate the proliferation and apoptosis processes in human retinoblastoma cells [44]. In module 2, ASCL4 was found to be essential for the determination of cell fate as well as the development and differentiation of numerous tissues [45].

In addition, we investigated the top 25 biological processes regulated by these TFs as shown in Supplementary Figure 1, where the percentage denotes the fraction of all TFs from the TF-miRNA coregulation network that were involved in the corresponding process. Consistent with the above observations, the TFs identified here are involved in a lot of OLP related processes, such as cell differentiation, Notch signaling pathway, steroid hormone mediated signaling pathway, and wound healing.

The analysis of TFs involved in TF-miRNA coregulation network indicates that these TFs regulate OLP related biological processes and play important roles in promoting the progression and development of OLP.

\section{Conclusion}

The potential malignant transformation of oral lichen planus (OLP) to oral cancer makes it demanding to understand the pathogenesis of this disease. In this paper, we introduced a novel approach to identify the TF-miRNA coregulation network that plays important roles in OLP. Unlike traditional approaches, the regulatory circuit we detected here provides new insights into observing disease associated patterns. The overlap between known OLP associated genes and our identified module genes implies that these gene modules are significantly related to OLP. The discriminative capacity of these modules in separating OLPs from controls confirms again the important roles of these modules in OLP and their potential as disease associated pattern. In addition, the regulators of these gene modules, including transcription factors and miRNAs, were also found to play important roles 

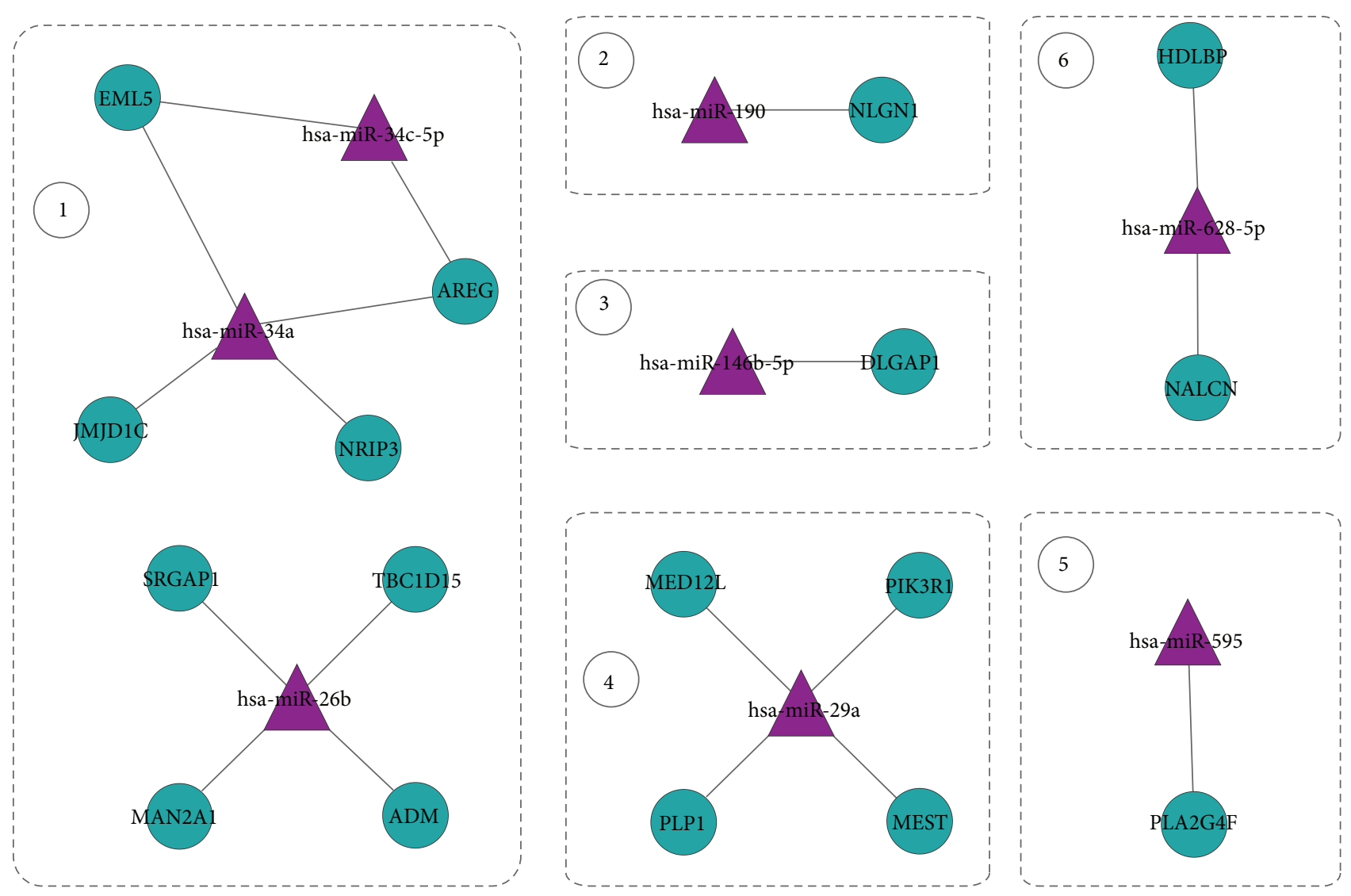

FIGURE 3: The regulatory relationship between miRNA and their targets in our regulatory network.

in the manifestation and progression of OLP, indicating their potential as new therapeutic targets when treating OLPs.

\section{Conflict of Interests}

The authors declare that there is no conflict of interests regarding the publication of this paper.

\section{Acknowledgments}

This work was partly supported by the guided project from Shanghai Science and Technology Commission (09411965900), the Natural Science Foundation of Shanghai Science and Technology Commission (14ZR1443600), the guided project from Shanghai Science and Technology Commission (14411971800), the Science Foundation of Shanghai Health Bureau (201440274), and the guided project from Science and Technology Department of Sichuan Province (2011SZ0300).

\section{References}

[1] S. Silverman Jr., "Oral lichen planus: a potentially premalignant lesion," Journal of Oral and Maxillofacial Surgery, vol. 58, no. 11, pp. 1286-1288, 2000.

[2] F. A. de Sousa, T. C. Paradella, A. A. H. Brandão, and L. E. B. Rosa, "Oral lichen planus versus epithelial dysplasia: difficulties in diagnosis," Brazilian Journal of Otorhinolaryngology, vol. 75, no. 5, pp. 716-720, 2009.

[3] P. B. Sugerman and N. W. Savage, "Oral lichen planus: causes, diagnosis and management," Australian Dental Journal, vol. 47, no. 4, pp. 290-297, 2002.

[4] A. Jemal, F. Bray, M. M. Center, J. Ferlay, E. Ward, and D. Forman, "Global cancer statistics," CA: A Cancer Journal for Clinicians, vol. 61, no. 2, pp. 69-90, 2011.

[5] T. Zheng, P. Boyle, H. Hu et al., "Dentition, oral hygiene, and risk of oral cancer: a case-control study in Beijing, People's Republic of China," Cancer Causes and Control, vol. 1, no. 3, pp. 235-241, 1990.

[6] M. Gorsky, J. B. Epstein, H. Hasson-Kanfi, and E. Kaufman, "Smoking habits among patients diagnosed with oral lichen planus," Tobacco Induced Diseases, vol. 2, no. 2, pp. 103-108, 2004.

[7] N. Mittal, G. S. Madhu Shankari, and S. Palaskar, "Role of angiogenesis in the pathogenesis of oral lichen planus," Journal of Oral and Maxillofacial Pathology, vol. 16, no. 1, pp. 45-48, 2012.

[8] M. Srinivasan, K. N. Kodumudi, and S. L. Zunt, "Soluble CD14 and toll-like receptor- 2 are potential salivary biomarkers for oral lichen planus and burning mouth syndrome," Clinical Immunology, vol. 126, no. 1, pp. 31-37, 2008.

[9] M. Ebrahimi, L. Boldrup, Y.-B. Wahlin, P. J. Coates, and K. Nylander, "Decreased expression of the p63 related proteins $\beta$ catenin, E-cadherin and EGFR in oral lichen planus," Oral Oncology, vol. 44, no. 7, pp. 634-638, 2008.

[10] X. A. Tao, C. Y. Li, J. Xia et al., "Differential gene expression profiles of whole lesions from patients with oral lichen planus," 
Journal of Oral Pathology and Medicine, vol. 38, no. 5, pp. 427433, 2009.

[11] X. Liu, Z.-P. Liu, X.-M. Zhao, and L. Chen, "Identifying disease genes and module biomarkers by differential interactions," Journal of the American Medical Informatics Association, vol. 19, no. 2, pp. 241-248, 2012.

[12] K.-Q. Liu, Z.-P. Liu, J.-K. Hao, L. Chen, and X.-M. Zhao, "Identifying dysregulated pathways in cancers from pathway interaction networks," BMC Bioinformatics, vol. 13, no. 1, article 126, 2012.

[13] G. A. Calin, C. Sevignani, C. D. Dumitru et al., "Human microRNA genes are frequently located at fragile sites and genomic regions involved in cancers," Proceedings of the National Academy of Sciences of the United States of America, vol. 101, no. 9, pp. 2999-3004, 2004.

[14] J. A. Chan, A. M. Krichevsky, and K. S. Kosik, "MicroRNA-21 is an antiapoptotic factor in human glioblastoma cells," Cancer Research, vol. 65, no. 14, pp. 6029-6033, 2005.

[15] S. M. Johnson, H. Grosshans, J. Shingara et al., "RAS is regulated by the let-7 microRNA family," Cell, vol. 120, no. 5, pp. 635-647, 2005.

[16] V. Gassling, J. Hampe, Y. Açil, J. H. Braesen, J. Wiltfang, and R. Häsler, "Disease-associated miRNA-mRNA networks in oral lichen planus," PLoS ONE, vol. 8, no. 5, Article ID e63015, 2013.

[17] R. Edgar, M. Domrachev, and A. E. Lash, "Gene Expression Omnibus: NCBI gene expression and hybridization array data repository," Nucleic Acids Research, vol. 30, no. 1, pp. 207-210, 2002.

[18] T. Nepusz, H. Yu, and A. Paccanaro, "Detecting overlapping protein complexes in protein-protein interaction networks," Nature Methods, vol. 9, no. 5, pp. 471-472, 2012.

[19] A. Krek, D. Grün, M. N. Poy et al., "Combinatorial microRNA target predictions," Nature Genetics, vol. 37, no. 5, pp. 495-500, 2005.

[20] B. John, A. J. Enright, A. Aravin, T. Tuschl, C. Sander, and D. S. Marks, "Human microRNA targets," PLoS Biology, vol. 2, no. 11, article e363, 2004.

[21] M. Maragkakis, P. Alexiou, G. L. Papadopoulos et al., "Accurate microRNA target prediction correlates with protein repression levels," BMC Bioinformatics, vol. 10, article 295, 2009.

[22] B. P. Lewis, C. B. Burge, and D. P. Bartel, "Conserved seed pairing, often flanked by adenosines, indicates that thousands of human genes are microRNA targets," Cell, vol. 120, no. 1, pp.1520, 2005.

[23] P. Sethupathy, B. Corda, and A. G. Hatzigeorgiou, “TarBase: a comprehensive database of experimentally supported animal microRNA targets," RNA, vol. 12, no. 2, pp. 192-197, 2006.

[24] L. Su, P. R. Morgan, and E. B. Lane, "Protein and mRNA expression of simple epithelial keratins in normal, dysplastic, and malignant oral epithelia," The American Journal of Pathology, vol. 145, no. 6, pp. 1349-1357, 1994.

[25] S. M. Xie, L. J. Shen, C. Yin, P. Ruan, and X. Yao, "Expression of tumor suppressor gene PTEN, PIP3 and cyclin D1 in oral squamous cell carcinoma and their correlations," Zhonghua Kou Qiang Yi Xue Za Zhi, vol. 41, no. 7, pp. 407-410, 2006.

[26] G. Brady, S. Crean, P. Naik, and S. Kapas, "Upregulation of IGF-2 and IGF-1 receptor expression in oral cancer cell lines," International Journal of Oncology, vol. 31, no. 4, pp. 875-881, 2007.

[27] M. J. Jameson, L. E. Taniguchi, K. K. Vankoevering et al., "Activation of the insulin-like growth factor-1 receptor alters p27 regulation by the epidermal growth factor receptor in oral squamous carcinoma cells," Journal of Oral Pathology \& Medicine, vol. 42, no. 4, pp. 332-338, 2013.

[28] D. W. Huang, B. T. Sherman, and R. A. Lempicki, "Bioinformatics enrichment tools: paths toward the comprehensive functional analysis of large gene lists," Nucleic Acids Research, vol. 37, no. 1, pp. 1-13, 2009.

[29] E. H. van der Meij, H. Mast, and I. van der Waal, “The possible premalignant character of oral lichen planus and oral lichenoid lesions: a prospective five-year follow-up study of 192 patients," Oral Oncology, vol. 43, no. 8, pp. 742-748, 2007.

[30] J. Vibha, K. Choudhary, M. Singh, M. Rathore, and N. Shekhawat, "A study on pharmacokinetics and therapeutic efficacy of Glycyrrhiza glabra a miracle medicinal herb," Botany Research International, vol. 2, pp. 157-163, 2009.

[31] C. M. Allen, F. M. Beck, K. M. Rossie, and T. J. Kaul, "Relation of stress and anxiety to oral lichen planus," Oral Surgery, Oral Medicine, Oral Pathology, vol. 61, no. 1, pp. 44-46, 1986.

[32] G. R. Ogden, A. Nairn, A. Carmichael et al., "Preservation of keratin expression in oral mucosa using a novel transport medium," Journal of Oral Pathology and Medicine, vol. 21, no. 1, pp. 17-20, 1992.

[33] S. Guo and L. A. Dipietro, "Factors affecting wound healing," Journal of Dental Research, vol. 89, no. 3, pp. 219-229, 2010.

[34] H. Kobayashi, K. Kumagai, A. Gotoh et al., "Upregulation of epidermal growth factor receptor 4 in oral leukoplakia," International Journal of Oral Science, vol. 5, no. 1, pp. 14-20, 2013.

[35] S. H. Günther, "Vitamin A acid in treatment of oral lichen planus," Archives of Dermatology, vol. 107, no. 2, p. 277, 1973.

[36] C.-Y. Lin, T.-S. Pan, C.-C. Ting et al., "Cytochrome P450 metabolism of betel quid-derived compounds: implications for the development of prevention strategies for oral and pharyngeal cancers," The Scientific World Journal, vol. 2013, Article ID 618032, 11 pages, 2013.

[37] E. Fernández-Miñano, C. Ortiz, A. Vicente, J. L. Calvo, and A. J. Ortiz, "Metallic ion content and damage to the DNA in oral mucosa cells of children with fixed orthodontic appliances," BioMetals, vol. 24, no. 5, pp. 935-941, 2011.

[38] K. Danielsson, M. Ebrahimi, Y. B. Wahlin, K. Nylander, and L. Boldrup, "Increased levels of COX-2 in oral lichen planus supports an autoimmune cause of the disease," Journal of the European Academy of Dermatology and Venereology, vol. 26, no. 11, pp. 1415-1419, 2012.

[39] N. A. Serrano, C. Xu, Y. Liu et al., "Integrative analysis in oral squamous cell carcinoma reveals DNA copy number-associated miRNAs dysregulating target genes," Otolaryngology-Head and Neck Surgery, vol. 147, no. 3, pp. 501-508, 2012.

[40] L. Cui, Y. Qi, H. Li et al., "Serum microRNA expression profile distinguishes enterovirus 71 and coxsackievirus 16 infections in patients with hand-foot-and-mouth disease," PLoS ONE, vol. 6, no. 11, Article ID e27071, 2011.

[41] Y. Li, C. Qiu, J. Tu et al., "HMDD v2.0: a database for experimentally supported human microRNA and disease associations," Nucleic Acids Research, vol. 42, no. 1, pp. D1070-D1074, 2014.

[42] M. Ding, J. Y. Xu, and Y. Fan, "Altered expression of mRNA for HIF- $1 \alpha$ and its target genes RTP801and VEGF in patients with oral lichen planus," Oral Diseases, vol. 16, no. 3, pp. 299-304, 2010.

[43] T. Wu, J. Jia, X. Xiong et al., "Increased expression of Lin28B associates with poor prognosis in patients with oral squamous cell carcinoma," PLoS ONE, vol. 8, no. 12, Article ID e83869, 2013. 
[44] S. W. Bai, B. Li, H. Zhang et al., "Pax6 regulates proliferation and apoptosis of human retinoblastoma cells," Investigative Ophthalmology and Visual Science, vol. 52, no. 7, pp. 4560-4570, 2011.

[45] M. Jonsson, E. Björntorp Mark, C. Brantsing, J. M. Brandner, A. Lindahl, and J. Asp, "Hash4, a novel human achaete-scute homologue found in fetal skin," Genomics, vol. 84, no. 5, pp. 859-866, 2004. 

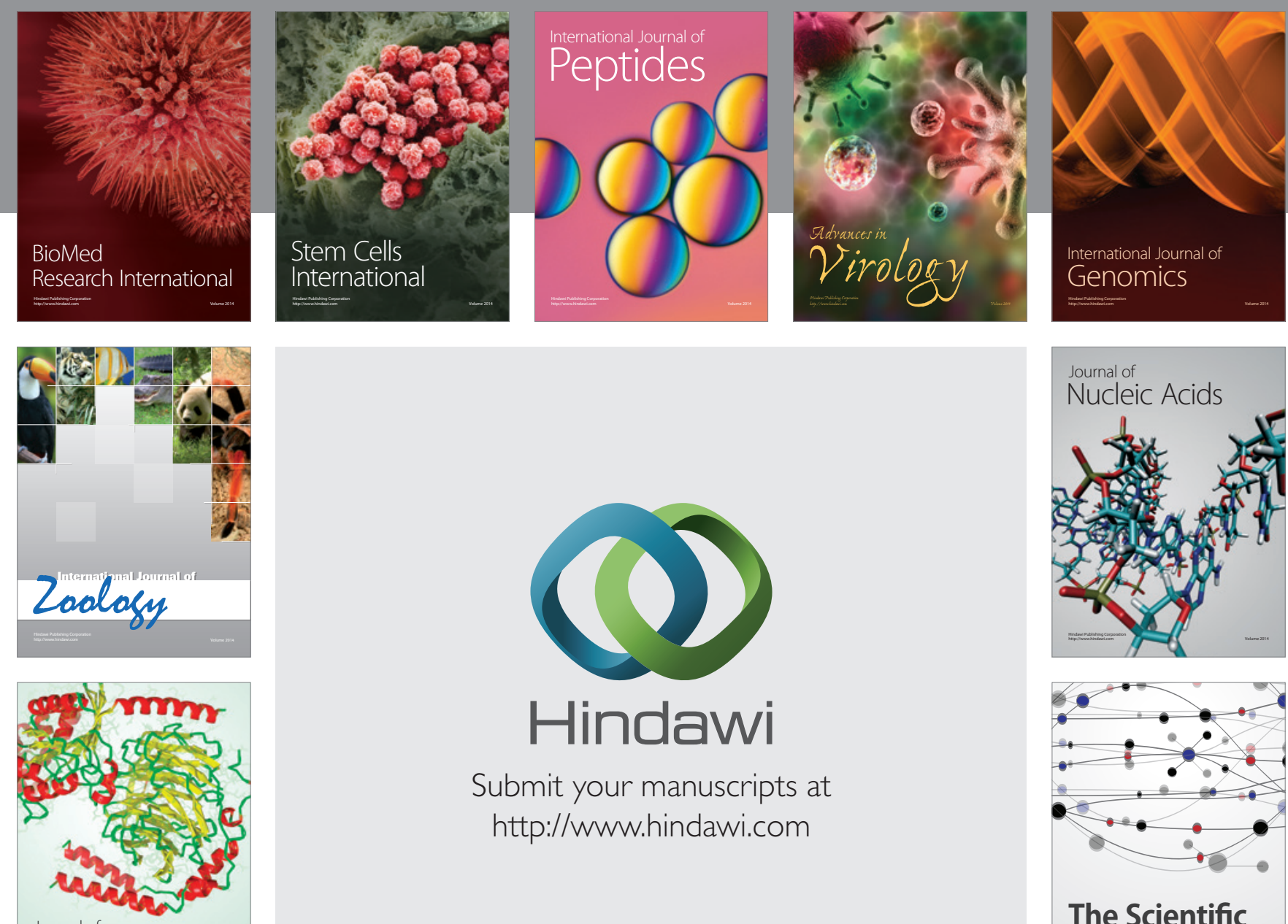

Submit your manuscripts at

http://www.hindawi.com

Journal of
Signal Transduction
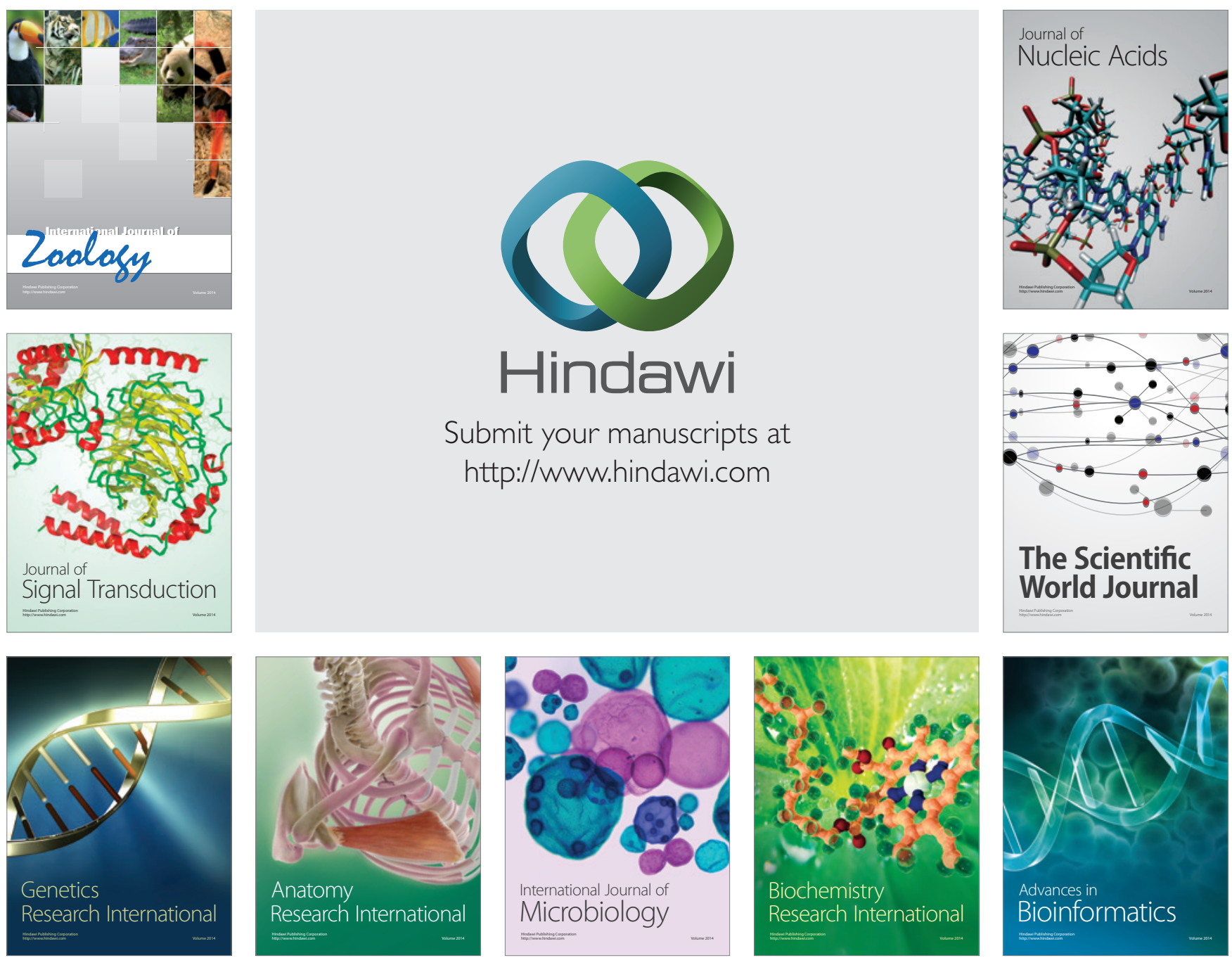

The Scientific World Journal
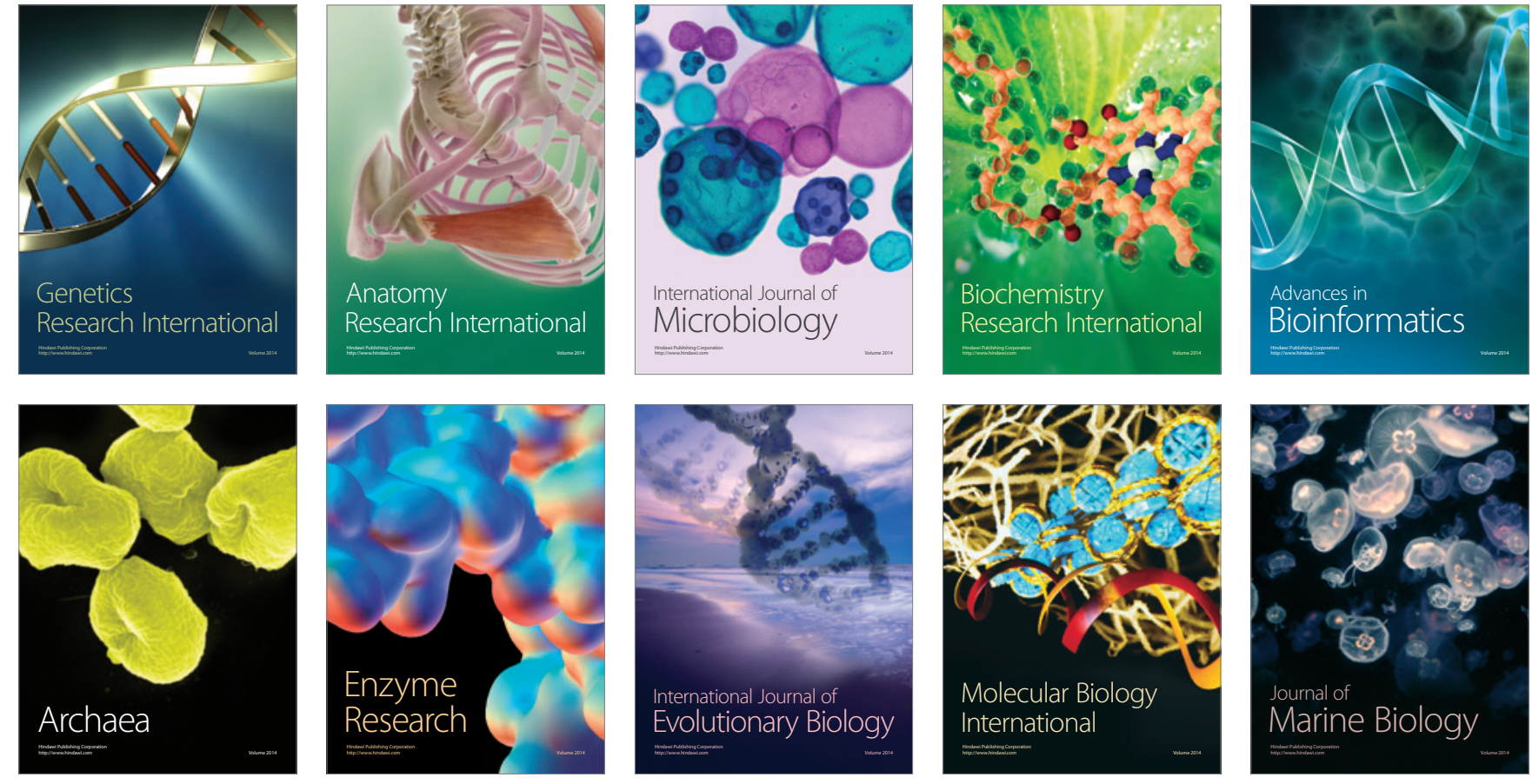